\title{
Productive Government Expenditure and Economic Performance in sub-Saharan Africa: An Empirical Investigation
}

\author{
Hammed Adetola Adefeso*
}

\begin{abstract}
This study examined the effect of government expenditure on its disaggregated level on economic growth in a sample of 20 sub-Saharan African Countries over the period of 19802010 in a dynamic panel data model. The result from Generalised Method of Moments (GMM) revealed an inverse relationship between productive government expenditure and economic growth in sub-Sahara Africa. Also, productive government expenditures were not actually productive most especially when financed by non-distortonary government tax revenue in sub-Saharan African countries. The study concluded that the productive government expenditure and its corresponding source of the mode of financing were counterproductive for economic performance in the African countries.
\end{abstract}

Keywords: Productive government expenditure; economic performance; GMM; non-distortionary taxation.

JEL Classification: $\mathrm{C} 01, \mathrm{H} 00$

\section{Introduction}

The empirical evidences on how government expenditure influences economic performance have remained contentious as results and evidence differ by country and region, analytical method employed and measure of public sector activity. The explanation provided to account for this inclusiveness in the literature can be grouped into different categories. The first category lies on the handful of studies (Helm, 1985; Kneller et al. 1999; 2001) which emphasised the failure of numerous researchers to take into consideration the implication of complete (full specification) government budget constraint in their regressions. Theoretically, government expenditure is

\footnotetext{
* Hammed Adetola Adefeso is at Department of Local Government Studies, Faculty of Administration, Obafemi Awolowo University, Ile-Ife, Nigeria
} 
classified into productive expenditure and unproductive expenditure which could be financed by different classifications of tax revenue. This lays emphasis on the need to consider both the sources and the uses of funds simultaneously for a meaningful evaluation of the effect of government expenditure or tax on growth otherwise the study is suffering from systematic biases to the parameter estimates associated with the implicit financing assumptions. Because of lack of a generally accepted theoretical framework that would pin down the most important determinants of growth whether government expenditure or not, the second category have emphasized the sensitivity of the findings to changes in the set of controls or conditioning variable used across studies (Levine-Renelt, 1992; Agell et. al. 1997), In addition, it is undoubted that there is dependence between government expenditure and the rate of growth (Wagner's Law) hence endogeneity problem as well as correlation of the government expenditure with the initial GDP (see Agell et al. 1997) which have not been sufficiently catered for.

In addition to this inconclusiveness of the growth effect of government expenditure, the existing studies have also displayed a disturbing trend as most of the literatures are either based on the developed countries or a large sample comprising of the mixture of the developed and developing countries. Despite the existence of a significant difference in the composition of government expenditure between developed and developing countries, not many studies have reported on the process by which government expenditure at its disaggregated level can shape the growth prospect of African countries. Specifically, out of thirty-six different empirical studies recognised by Kneller et al. (1998) as the main studies on fiscal policy variables and growth relationship, only three studies are from developing countries and no single study was reported based on panel data analysis from sub-Saharan Africa. Another justification for limiting the sample to sub-Saharan Africa is the wide spread perception that the region is structurally different from the rest of the world because they share key economic and cultural characteristic and thus provide homogeneity in the group of countries selected for the study. Infact, many policymakers from Africa believe that the lesson from Latin America or East Asia do not apply to them simply because they have different economic environment and this enable them to lean from one another. Therefore, an empirical analysis that focuses on the growth performance effect of disaggregated government expenditure within sub-Saharan African countries will have greater credibility among policymaker from Africa.

The primary objective of this study is to examine the growth effect of productive government expenditure for a panel of 20 sub-Saharan African countries paying attention to the sensitivity issue arising from initial condition and conditioning variables. Concerning the problem of potential endogeneity as a result of Wagner's Law, this study employs Generalised Methods of Moment (GMM) estimation method of analysis. The study also pays attention to the possible omitted variable bias we just mentioned by taking into consideration complete implication of government budget 
constraint and the potential collinearity between the government expenditure and tax components. The rest of the paper is structures as follows: section 2 consists of predictions of endogenous growth models and literature review, section 3 comprises of methodology and the main findings from the study and summary $\&$ conclusion are drawn from section 4 .

\section{Prediction of Endogenous Growth Model}

In the standard neoclassical growth models (like Judd, 1985; Chamley, 1986), the steady-state growth rate is undoubtedly determined by the exogenous growth of labour supply and technical progress. Hence, the models are unable to explain the growth effects of fiscal variables as the fiscal policy can only influence the transition path to the steady-state. However, the most recent endogenous growth models (like Barro, 1990; Barro and Sala-i-Martin, 1992; 1995, Mendoza et.al. 1997) attempt to transform the neoclassical temporary growth effects of fiscal policy into permanent growth effects by providing mechanisms through which fiscal policy affect the level of output and the steady-state growth rate.

Endogenous growth models classify fiscal policy variables into four categories: productive or unproductive government expenditure and distortionary or non-distortionary taxation. Government expenditures that allowed entering into private production function as a variable affects the marginal product of private capital and hence boost growth rate is considered as productive. If otherwise, then they are classified as unproductive government expenditure which do not influence growth rate. Distortionary taxation affects saving/investment decisions of the private agent by weaken the incentive to invest in physical/human capital and hence distorts the growth process of the steady-state but if otherwise, it is classified as non-distortionary taxation which does not affect growth rate because of the assumed nature of the private agents' utility function.

A simple model of endogenous growth such as Barro (1990) where the interaction between private and public capital is elegantly captured therefore, predict that the growth effect of non-distortionary tax and productive expenditure is positive while that of distortionary tax and unproductive expenditure is negative. An increase in productive government expenditure financed by non-distortionary taxation is predicted to have growth-enhancing effect but with the distortionary tax the growth effect is ambiguous. Similarly, an increase in non-productive expenditure financed by non-distortionary tax is predicted to have zero growth effect whereas growth effect is negative if distortionary tax is used.

The importance of the correct specification of the government budget constraint in analysis of the growth effect of the fiscal policy has been extensively discussed by 
Kneller et al. (1999) for the developed economies. They noted that most researchers actually estimated eq.(1) which does not take into consideration implicit financing element:

$$
\mathrm{g}_{\mathrm{it}}=\alpha+\sum_{i=1}^{k} \delta_{i} C_{i t}+\sum_{j=1}^{n} \sigma_{j} F_{j t}+\mu_{i t}
$$

This is possible because as rightly noted in the study of Tanzi and Zee (1997), numerous researchers have used any of the three indicators of fiscal policy (government expenditure, tax revenue, budget deficit/surplus) to measure the stance of fiscal policy. However, Levine-Renelt (1992) find that none of these fiscal indicators is robustly correlated with growth rate of the economy when evaluated individually and this accounts for the wide spread of the non-robustness of the studies on the growth effect of the fiscal policy variables across studies. Since in theory, if the budget constraint is completely specified i.e. expenditure equals revenue then $\sum_{j=1}^{n} F_{j t}=0$.

$$
\mathrm{g}_{\mathrm{it}}=\alpha+\sum_{i=1}^{k} \delta_{i} C_{i t}+\sum_{j=1}^{n-1} \sigma_{j} F_{j t}+\sigma_{n} F_{n t}+\mu_{i t}
$$

Purposely to avoid the perfect collinearity in the fiscal instruments arising from the identity of the complete government budget constraint, Kneller et al. (1999) simply omits $F_{n t}$ (the one with neutral effect on growth as suggested by theory, $\sigma_{n}=0$ ) and concluded that the correct specification of the equation to be estimated on growth effect of fiscal policy is of the form:

$$
\mathrm{g}_{\mathrm{it}}=\alpha+\sum_{i=1}^{k} \delta_{i} C_{i t}+\sum_{j=1}^{n-1}\left(\sigma_{j}-\sigma_{n}\right) F_{j t}+\mu_{i t}
$$

Where $\mathrm{g}_{\mathrm{it}}=$ growth rate of country $\mathrm{i}$ at time $\mathrm{t}, \alpha=$ constant term, $C_{i t}=$ conditioning (non-fiscal) variables, $\delta_{i}=$ slope of the conditioning variables, $F_{j t}=$ fiscal variables, $\sigma_{j}=$ coefficient that measure the growth impact of the variable $F_{j t}$, one of $n-1$ fiscal variables, $\sigma_{n}=$ coefficient that measure the growth effect of the fiscal $n$th instrument which is use to fund change in one of the $n-1$ fiscal policy variables. The hypothesis test of $\sigma_{j}=0$ conducted in empirical studies is in actual fact testing the null hypothesis that $\left(\sigma_{j}-\sigma_{n}\right)=0$ and not the former as implicitly assumed i.e. the studies actually estimate the impact of a change in one fiscal variable when there is an offsetting change in the omitted $n$th fiscal instrument, which implicitly finances the variation in the variable of interest. Therefore, the correct coefficient of fiscal structure is $\sigma_{j}-\sigma_{n}$ which captured the implicit financing element and it is interpreted as the effect of a unit change in the relevant variable offset by a unit change in the omitted category. Equation 3 is the estimable model for this study as explained by Kneller et al. (1999), Amanja and Morrissey (2005), Adefeso et al (2010) as cited in Ahmad and Wajid (2013) 


\section{A Review of Empirical Evidence on Fiscal Policy-Economic Growth Nexus}

The empirical findings on fiscal policy-economic growth nexus is presented in table 1 to table 4 below. This study follows Kneller et al. (1999) by categorising the empirical findings into the following headings:

(i) Empirical evidences on Tax Revenue and Economic Growth

(ii) Empirical evidences on Government consumption expenditure and economic growth

(iii) Empirical evidences on Transfer payment or welfare expenditure and economic growth

(iv) Empirical evidences on Public investment expenditure and economic growth

Table 1: Empirical evidences on tax revenue and economic growth

\begin{tabular}{|c|c|c|c|c|c|}
\hline Author & Countries & Years & $\begin{array}{l}\text { Econometric } \\
\text { Method }\end{array}$ & $\begin{array}{l}\text { Length of } \\
\text { Average }\end{array}$ & Main results \\
\hline Marsden (1983) & $\begin{array}{l}10 \text { pairs of } \\
\text { Matched GDP }\end{array}$ & $1970 \mathrm{~S}$ & $\begin{array}{l}\text { Pair } \\
\text { Comparisons }\end{array}$ & & Low tax countries grew quicker than tax countries \\
\hline $\begin{array}{l}\text { Koester, } \\
\text { Kormendi (1989) }\end{array}$ & 63 & 1970-1979 & Cross-section & 10-years & $\begin{array}{l}\text { Marginal tax and average tax rates have no significant } \\
\text { negative effect }\end{array}$ \\
\hline Skinner (1987) & $\begin{array}{l}\text { African } \\
\text { countries }\end{array}$ & & Cross-section & & $\begin{array}{l}\text { Income, corporation and import taxes are significant and } \\
\text { negative. Export and sales taxes insignificant }\end{array}$ \\
\hline $\begin{array}{l}\text { Engen, Skinner } \\
(1992)\end{array}$ & 107 & $1970-1985$ & Cross-section & 16years & $\begin{array}{l}\text { Taxes have significant and negative effects in short and } \\
\text { long-run. }\end{array}$ \\
\hline Dowrick (1992) & OECD & $1960-1985$ & Cross-section & 26years & $\begin{array}{l}\text { Income taxes significant negative Corporation taxes not } \\
\text { significant. }\end{array}$ \\
\hline $\begin{array}{l}\text { Easterly, Rebelo } \\
(1993)\end{array}$ & 100 & 1970-1988 & Cross-section & 19years & $\begin{array}{l}\text { Income taxes significant and negative. other type of } \\
\text { taxation non-robust. }\end{array}$ \\
\hline Cashin (1995) & $23 \mathrm{OECD}$ & $1971-88$ & Panel & 5 -years & Total taxation significant negative \\
\hline $\begin{array}{l}\text { Mendoza, milesi } \\
\text { ferreti, Asea } \\
(1996)\end{array}$ & II OECD & $1965-91$ & Panel & $\begin{array}{l}\text { Annual, } \\
5 \text {-year }\end{array}$ & $\begin{array}{l}\text { Effective capital, consumption and labour tax rates are } \\
\text { insignificant in 5-years averages non-robustly significant in } \\
\text { annual data regressions. }\end{array}$ \\
\hline $\begin{array}{l}\text { Yi, Kocherlakota } \\
(1996)\end{array}$ & US UK & $\begin{array}{l}\text { US1891- } \\
\text { 1991-UK } \\
1831-1991\end{array}$ & Time-series & $\begin{array}{l}\text { Annual (10 } \\
\text { lags) }\end{array}$ & $\begin{array}{l}\text { Tax measures insignificant individually, significant when } \\
\text { put with public capital term. }\end{array}$ \\
\hline Agell et al.(1997) & $23 \mathrm{OECD}$ & $1970-1990$ & & 21years & $\begin{array}{l}\text { Initially, the average share of tax revenues in GDP is } \\
\text { negatively correlated with average annual growth rate but } \\
\text { turned positive when initial GDP per capital and share } \\
\text { of population younger than } 15 \text { and older than } 65 \text { were } \\
\text { included as explanatory variables. }\end{array}$ \\
\hline Poot (2000) & & 1983-1998 & & 16years & $\begin{array}{l}\text { The study found empirical support for the negative effect } \\
\text { of taxes on growth }\end{array}$ \\
\hline $\begin{array}{l}\text { Adefeso et al. } \\
(2010)\end{array}$ & Nigeria & $1970-2005$ & Time-series & 35years & $\begin{array}{l}\text { Non-distortionary taxation and non-productive } \\
\text { expenditures had neutral impact on economic growth. }\end{array}$ \\
\hline $\begin{array}{l}\text { Babalola and } \\
\text { Aminu (2011) }\end{array}$ & Nigeria & 1977-2009 & Time-series & 32years & $\begin{array}{l}\text { Distortionary revenue and economic growth were } \\
\text { positively related }\end{array}$ \\
\hline
\end{tabular}

Source: Kneller et al (1998) Modified 
Table 2: Empirical evidences on government consumption expenditure and economic growth

\begin{tabular}{|c|c|c|c|c|c|}
\hline Author & Countries & Years & $\begin{array}{c}\text { Econometric } \\
\text { Method }\end{array}$ & $\begin{array}{l}\text { Length of } \\
\text { Average }\end{array}$ & Main results \\
\hline Landau (1983) & 104 & $1961-76$ & Cross-section & 16years & $\begin{array}{l}\text { Government consumption expenditure has a } \\
\text { significant negative effect. }\end{array}$ \\
\hline $\begin{array}{l}\text { Kormendi, } \\
\text { Meguire (1985) }\end{array}$ & 47 & & Cross-section & 28years & $\begin{array}{l}\text { Government consumption expenditure has a } \\
\text { no significant effect. }\end{array}$ \\
\hline Ram (1986) & 115 & $1960-80$ & $\begin{array}{l}\text { Cross-section } \\
\text { Time series }\end{array}$ & 10 & $\begin{array}{l}\text { Size of government produces significant } \\
\text { positive coefficients }\end{array}$ \\
\hline Landau (1986) & LDCs & & Cross-section & & $\begin{array}{l}\text { Government consumption expenditure has a } \\
\text { significant negative effect }\end{array}$ \\
\hline $\begin{array}{l}\text { Grier, Tullock } \\
(1989)\end{array}$ & 115 & $1950-81$ & Panel data & 5-years & $\begin{array}{l}\text { Government consumption expenditure has a } \\
\text { significant negative effect }\end{array}$ \\
\hline Romer (1989a) & 94 & $1960-85$ & Cross-section & 16 years & $\begin{array}{l}\text { Government consumption expenditure has a } \\
\text { significant positive effect }\end{array}$ \\
\hline Romer (1989b) & 112 & $1960-85$ & Cross-section & 16years & $\begin{array}{l}\text { Government consumption expenditure has a } \\
\text { significant positive effect }\end{array}$ \\
\hline Romer (1990) & 90 & $1969-85$ & Cross-section & 16 years & $\begin{array}{l}\text { Government consumption expenditure has a } \\
\text { significant positive effect }\end{array}$ \\
\hline $\begin{array}{l}\text { Alexander } \\
(1990)\end{array}$ & 13 OECD & 1959-84 & Panel & Annual & $\begin{array}{l}\text { Government consumption expenditure has a } \\
\text { significant negative effect }\end{array}$ \\
\hline Barro (1991) & 98 & $1960-85$ & Cross-section & 16 years & $\begin{array}{l}\text { Government consumption expenditure has a } \\
\text { significant negative effect }\end{array}$ \\
\hline Lin (1994) & 62 countries & $1960-1985$ & Panel & 26years & $\begin{array}{l}\text { Mixed results, government consumption } \\
\text { expenditure not significant in developed } \\
\text { countries but significantly positive in less } \\
\text { developed countries. }\end{array}$ \\
\hline $\begin{array}{l}\text { Kneller et al. } \\
(1999)\end{array}$ & $\begin{array}{l}22 \text { OECD } \\
\text { Countries }\end{array}$ & 1970-1995 & Panel & 26years & $\begin{array}{l}\text { Government consumption expenditure does } \\
\text { not enhance growth }\end{array}$ \\
\hline $\begin{array}{l}\text { Dunne and } \\
\text { Nikolaidou } \\
(1999)\end{array}$ & Greece & 1960-1996 & Time series & 37years & $\begin{array}{l}\text { Government consumption expenditure does } \\
\text { not affect growth }\end{array}$ \\
\hline Tanninen (1999) & 52 countries & 1970-1992 & Panel & 23years & $\begin{array}{l}\text { Government consumption expenditure has } \\
\text { negative impact }\end{array}$ \\
\hline Poot (2000) & & 1983-1998 & & 16years & $\begin{array}{l}\text { He did not find conclusive evidence for } \\
\text { the relationship between government } \\
\text { consumption and growth. }\end{array}$ \\
\hline $\begin{array}{l}\text { Gupta et al. } \\
(2005)\end{array}$ & $\begin{array}{l}39 \text { Lower } \\
\text { income } \\
\text { countries }\end{array}$ & $1990-2002$ & Panel & 13years & $\begin{array}{l}\text { Countries where Spending is concentrated } \\
\text { on wages tend to have lower growth. }\end{array}$ \\
\hline
\end{tabular}

Source: Kneller et al (1998) Modified 
Table 3: Empirical evidences on transfer payment/welfare expenditure and economic growth

\begin{tabular}{|c|c|c|c|c|c|}
\hline Author & Countries & Years & $\begin{array}{c}\text { Econometric } \\
\text { Method }\end{array}$ & $\begin{array}{l}\text { Length of } \\
\text { Average }\end{array}$ & Main results \\
\hline Landau (1983) & & & & & $\begin{array}{l}\text { Transfer payment expenditure has no } \\
\text { significant effect. }\end{array}$ \\
\hline Korpi (1985) & OECD & 1970-87 & Panel & 18 years & $\begin{array}{l}\text { Transfer payment expenditure has a } \\
\text { significant negative effect }\end{array}$ \\
\hline Landau (1985) & 16 OECD & $1952-76$ & Panel/ cross -section & Annual & $\begin{array}{l}\text { Transfer payment expenditure has no } \\
\text { significant effect. }\end{array}$ \\
\hline Weede (1986) & 19 OECD & $1960-82$ & Panel/cross-section & 7-years & $\begin{array}{l}\text { Transfer payment expenditure has a } \\
\text { significant positive effect }\end{array}$ \\
\hline $\begin{array}{l}\text { McCallum, Blais } \\
(1987)\end{array}$ & 17 OECD & $1960-83$ & Panel/cross-section & 7-years & $\begin{array}{l}\text { Transfer payment expenditure has a } \\
\text { significant negative effect }\end{array}$ \\
\hline $\begin{array}{l}\text { Castles,Dowrick } \\
\text { (1990) }\end{array}$ & 18 OECD & $1960-85$ & Panel & 6 years & $\begin{array}{l}\text { Transfer payment expenditure has a } \\
\text { significant negative effect }\end{array}$ \\
\hline Weede (1991) & 19 OECD & $1960-85$ & Panel & 7-years & $\begin{array}{l}\text { Transfer payment expenditure has a } \\
\text { significant positive effect }\end{array}$ \\
\hline Nordstrum (1992) & 14 OECD & $1970-89$ & Cross-section & 20 years & $\begin{array}{l}\text { Transfer payment expenditure has a } \\
\text { significant positive effect }\end{array}$ \\
\hline $\begin{array}{l}\text { Sala-i-Marin } \\
\text { (1992) }\end{array}$ & 75 & & Cross-section & & $\begin{array}{l}\text { Transfer payment expenditure has a } \\
\text { significant positive effect }\end{array}$ \\
\hline $\begin{array}{l}\text { Person, Tabellini } \\
\text { (1994) }\end{array}$ & 14 OECD & $1960-85$ & Cross-section & 16 years & $\begin{array}{l}\text { Transfer payment expenditure has a } \\
\text { significant positive effect }\end{array}$ \\
\hline $\begin{array}{l}\text { Hanson, } \\
\text { Henrekson(1994) }\end{array}$ & $\begin{array}{l}14 \text { industries } \\
\text { for OECD }\end{array}$ & 1970-1987 & Cross- section & 18 years & $\begin{array}{l}\text { Transfer payment expenditure has no } \\
\text { significant effect. }\end{array}$ \\
\hline Cashin (1995) & 23OECD & 1971-1988 & Panel & 5-years & $\begin{array}{l}\text { Transfer payment expenditure has a } \\
\text { significant positive effect }\end{array}$ \\
\hline $\begin{array}{l}\text { Nazmi, Ramirez } \\
\text { (1997) }\end{array}$ & Mexico & $1950-1990$ & Time-series & Annual & $\begin{array}{l}\text { Transfer payment expenditure has a } \\
\text { significant positive effect }\end{array}$ \\
\hline
\end{tabular}

Source: Kneller et al (1998) Modified 
Table 4: Public investment expenditure and growth studies

\begin{tabular}{|c|c|c|c|c|c|}
\hline Author & Countries & Years & $\begin{array}{c}\text { Econometric } \\
\text { Method }\end{array}$ & $\begin{array}{l}\text { Length of } \\
\text { Average }\end{array}$ & Main results \\
\hline Landau(1986) & LDCs & & & & Education, defence, capital Expenditure insignificant. \\
\hline $\begin{array}{l}\text { Barth,Bradley } \\
\text { (1988) }\end{array}$ & 16 OECD & 1971-83 & Cross-section & 13years & $\begin{array}{l}\text { Total public investment } \\
\text { Insignificant. }\end{array}$ \\
\hline Barro (1989) & 72 & \begin{tabular}{|l|}
$1960-85$ \\
\end{tabular} & \begin{tabular}{|l|} 
Cross-section \\
\end{tabular} & 16 years & Total investment significant \\
\hline Barro (1991) & 98 & $1960-85$ & Cross-section & 16 years & $\begin{array}{l}\text { Transport and communication significant.total public } \\
\text { investment insignificant. }\end{array}$ \\
\hline $\begin{array}{l}\text { Easterly, rebelo } \\
\text { (1993) }\end{array}$ & 100 & $1970-88$ & Cross-section & 19 years & $\begin{array}{l}\text { Transport and communication significant. Total } \\
\text { investment education,health insignificant. }\end{array}$ \\
\hline $\begin{array}{l}\text { Devarajan et al. } \\
\text { (1993) }\end{array}$ & 14 OECD & $1970-1988$ & Panel & 19years & $\begin{array}{l}\text { Government expenditure on health and transport and } \\
\text { communications are growth promoting but found no } \\
\text { positive impact of education and military spending. }\end{array}$ \\
\hline $\begin{array}{l}\text { Hansson, } \\
\text { Henrekson } \\
(1994)\end{array}$ & & & & & Education spending is positive \\
\hline $\begin{array}{l}\text { Devarajan, } \\
\text { Swaroop, } \\
\text { Zou (1996) }\end{array}$ & $\begin{array}{l}14 \\
\text { developed } \\
\text { countries } \\
\end{array}$ & $1970-1990$ & Panel & $\begin{array}{l}\text { 5-years } \\
\text { moving } \\
\text { Average }\end{array}$ & $\begin{array}{l}\text { Health transport and communication significant positive, } \\
\text { defence, education significant negative. }\end{array}$ \\
\hline $\begin{array}{l}\text { Devarajan et al. } \\
\text { (1996) }\end{array}$ & $\begin{array}{l}43 \\
\text { Developing } \\
\text { countries }\end{array}$ & $1970-1990$ & & 21years & $\begin{array}{l}\text { Capital expenditures have significant and negative } \\
\text { impact on economic growth whereas current } \\
\text { (unproductive) expenditures have positive and } \\
\text { significant influence on economic growth. The } \\
\text { negative impact of capital expenditures is due to } \\
\text { excessive government expenditures towards productive } \\
\text { expenditures at the expense of non-productive } \\
\text { expenditure }\end{array}$ \\
\hline $\begin{array}{l}\text { Yi, } \\
\text { Kocherlakotas } \\
(1996)\end{array}$ & \begin{tabular}{|l} 
US UK \\
\end{tabular} & $\begin{array}{l}\text { US 1891- } \\
1991 \\
\text { UK 1831- } \\
1991\end{array}$ & Time-series & $\begin{array}{l}\text { Annual } \\
\text { (10 lags) }\end{array}$ & $\begin{array}{l}\text { Public investment insignificant when included } \\
\text { individually. significant when included with tax } \\
\text { variables }\end{array}$ \\
\hline $\begin{array}{l}\text { Kneller et } \\
\text { al.(1999) }\end{array}$ & $\begin{array}{l}22 \text { OECD } \\
\text { Countries }\end{array}$ & $1970-95$ & Panel & 26years & Government investment enhances growth \\
\hline $\begin{array}{l}\text { Dunne and } \\
\text { Nikolaidou } \\
(1999)\end{array}$ & Greece & $1960-96$ & Time series & 37years & $\begin{array}{l}\text { Military/defence expenditure have a negative effect on } \\
\text { growth }\end{array}$ \\
\hline Tanninen (1999) & 52 countries & $1970-92$ & Panel & 23years & $\begin{array}{l}\text { Large government spending on public goods is growth } \\
\text { retarding but not for small government spending, social } \\
\text { security spending is positive }\end{array}$ \\
\hline Poot (2000) & & $1983-98$ & & 16years & $\begin{array}{l}\text { There is positive link between growth and education } \\
\text { spending while the evidence on the negative growth } \\
\text { impact of defense spending is moderately strong, } \\
\text { also non-robust positive associations exist between } \\
\text { infrastructure spending and economic growth. }\end{array}$ \\
\hline $\begin{array}{l}\text { Albala and } \\
\text { Mamatzakis } \\
(2001)\end{array}$ & Chile & 1960-95 & Time series & 36years & $\begin{array}{l}\text { Positive and significant correlation between public } \\
\text { infrastructure and economic growth. }\end{array}$ \\
\hline
\end{tabular}




\begin{tabular}{|c|c|c|c|c|c|}
\hline $\begin{array}{l}\text { Bleaney et al. } \\
(2001)\end{array}$ & & $1970-95$ & Panel & $\begin{array}{l}\text { 5years } \\
\text { averaging }\end{array}$ & $\begin{array}{l}\text { Results completely support Barro's prediction. } \\
\text { Education and health }\end{array}$ \\
\hline $\begin{array}{l}\text { Milbourne, Otto } \\
\text { and Voss (2003) }\end{array}$ & $\begin{array}{l}74 \text { industrial } \\
\text { and } \\
\text { developing } \\
\text { countries } \\
\end{array}$ & & & & Positive correlation between investment and growth \\
\hline Yasin (2003) & \begin{tabular}{|l|} 
Sub- \\
Saharan \\
Africa \\
\end{tabular} & & Panel & & $\begin{array}{l}\text { Government spendings on capital formation have } \\
\text { positive and significant effect on growth. }\end{array}$ \\
\hline Derin (2003) & $\begin{array}{l}33 \\
\text { developing } \\
\text { countries, } \\
15 \\
\text { European } \\
\text { Union } \\
\text { countries }\end{array}$ & $1970-99$ & Panel & $\begin{array}{l}\text { 20years, } \\
\text { 5years } \\
\text { averaging } \\
\text { data }\end{array}$ & $\begin{array}{l}\text { Investment and per capital GDP are positively and } \\
\text { significantly related. Distortionary taxation has negative } \\
\text { and significant impact in EU countries while it has } \\
\text { insignificant relation in case of developing countries. } \\
\text { Distortionary tax does not enhance long run growth } \\
\text { in developing economies. Productive expenditures } \\
\text { have negative and significant impact in developing } \\
\text { countries while it has insignificant relation in case of } \\
\text { EU countries. Endogenous growth model holds for } \\
\text { only developing countries. The impact of labour force } \\
\text { growth, non-distortionary taxation and non-productive } \\
\text { expenditures on long run per capital GDP is insignificant } \\
\text { for both the developed and developing countries. }\end{array}$ \\
\hline Benos (2004) & $\begin{array}{l}16 \text { OECD } \\
\text { countries }\end{array}$ & $1990-97$ & Panel & 8years & $\begin{array}{l}\text { Inverted U-shaped relation of health, education and fuel } \\
\text { energy expenditures with economic growth. Education } \\
\text { has a strong positive relation to economic growth for } \\
\text { poor countries and health expenditures have an inverse } \\
\text { relation to it. There exist a U-Shaped relationship of } \\
\text { housing, transport, communication, social security } \\
\text { expenditures with economic growth. }\end{array}$ \\
\hline $\begin{array}{l}\text { Gupta et al. } \\
(2005)\end{array}$ & $\begin{array}{l}39 \text { Lower } \\
\text { income } \\
\text { countries }\end{array}$ & 1990-02 & Panel & 13years & $\begin{array}{l}\text { If the structure of the government expenditures consists } \\
\text { of more productive than non-productive expenditure } \\
\text { then it has positive impact on economic growth. } \\
\text { Those countries that allocate higher shares to capital } \\
\text { and nonwage goods and services enjoy faster output } \\
\text { expansion. }\end{array}$ \\
\hline $\begin{array}{l}\text { Amanja and } \\
\text { Morrissey } \\
(2005)\end{array}$ & Kenya & 1964-04 & Time-series & 40years & $\begin{array}{l}\text { The results were contrary to the public policy } \\
\text { endogenous growth model that distortionary taxation } \\
\text { promotes economic growth and productive expenditures } \\
\text { stifle it. Non-productive and non-distortionary taxation } \\
\text { had neutral impact on economic growth which is } \\
\text { consistent with Barro's prediction. }\end{array}$ \\
\hline Kukk (2007) & & & Cross- section & & $\begin{array}{l}\text { Productive and non-productive expenditures had } \\
\text { positive and negative impact on economic growth } \\
\text { respectively }\end{array}$ \\
\hline $\begin{array}{l}\text { Adefeso et } \\
\text { al.(2010) }\end{array}$ & Nigeria & $1970-05$ & Time-series & 35years & $\begin{array}{l}\text { Productive expenditures had positive impact on } \\
\text { economic growth. }\end{array}$ \\
\hline $\begin{array}{l}\text { Babalola and } \\
\text { Aminu (2011) }\end{array}$ & Nigeria & 1977-09 & Time-series & 32years & $\begin{array}{l}\text { Productive expenditures and economic growth were } \\
\text { positively and significantly associated in the long run } \\
\text { but positive and insignificant in the short run }\end{array}$ \\
\hline
\end{tabular}

Source: Kneller et al (1998) Modified 


\section{Data Description, Theoretical Classification of Fiscal Variables and Research Methodology}

\section{Data Description, Theoretical Classification of Fiscal Variables}

Barro (1990) classifies the government expenditures based on their impact on economic growth as productive government expenditures which have a positive impact on economic growth, unproductive government expenditures which are neutral or have an insignificant impact on economic growth, and other public expenditures which have an insignificant impact on economic growth. Similarly, government tax revenues have been classified as distortionary taxation which its economic growth effect is negative, non-distortionary which its economic growth effect neutral or insignificant and others tax revenues which have insignificant effect. These classifications are summarized in the study of Kneller et al (1998), Benos (2004) among others and the vector $C_{i t}$ consists of a set of variables identified by Levine and Renelt (1992) as the important control variables for cross-country growth regressions such as average real investment share of GDP proxied by capital formation/ investment in physical capital, human capital proxied by secondary school enrolment rate and average growth rate of the population which were all sourced from World Development Indicator (WDI).

\section{Econometric Models and Analysis of Results}

The study departs a little from the line of the prediction of the public policy endogenous growth models as employed by Kneller et al. (1999; 2001) who follow Barro (1990), complete specification of government budget constraints are unable to be taken into consideration in full because of limitation of data availability most especially at their disaggregated level in Africa. This study only takes into consideration both productive government expenditure and non distortionary government tax revenue. The study recognised the submission of Tanzi and Zee (1997) that are three indicators of fiscal policy and these are government expenditures, taxes and deficit. Many researchers have used government expenditure to measure the stance of fiscal policy like Barro (1999), Barro and Sala-i-martin (1995), Ambler and Paquet (1996) among others. Other authors have used tax rates see for example, Engen and Skinner (1996), Stokely and Rebelo (1995), Xu (1994) or deficit measures like the study of Easterly and Rebelo (1993). Levine and Renelt (1992) found that none of these fiscal indicators is robustly correlated with economic growth when evaluated individually. Both the source of the revenues and expenses must be taken into consideration for meaningful evaluation on any Fiscal Policy variable-Economic Growth nexus. This has led to the formulation of 3 variant models of equation 3. This study imposed zero restriction 
on budget deficit which is presented in the model I and omits government revenue in the model II while in model III, government expenditure is omitted. In Model I however, in order to avoid perfect multicollinearity only budget deficit was omitted in the model. Theoretically, model I yields more precise and accurate measures of fiscal policy variables with lower standard errors because both the source of the revenue and expenditure are taken into consideration. Theoretical aggregation of functional classification of disaggregated fiscal policy variables is well documented in the studies of Benos (2004); Kneller (1999) among others.

In other to control the endogeneity problem raised in section one of this study, instrumental variable (IV) methods are employed in the empirical estimations. This IV methods employed will solve the simultaneity bias between regressors and regressand and the error measurement. The application of the Generalised Method of Moments (GMM) technique has been recognised as an extension of IV method of estimation which uses predetermined values of the right-hand side variables as instruments. The empirical findings of Knerller et al. (1999) and Brons et al. (2000) recommend GMM estimation on which is equally supported by the theory.

\section{Econometric Models}

In line with the theoretical background of this study, the dynamic behaviour of the economic relationships being studied is achieved by estimating a dynamic panel regression model specified as follows:

$$
g_{i t}=\sigma g_{i t-1}+\beta X_{i t}^{\prime}+\mu_{i}+v_{i t}
$$

Where $g_{i t}$ represents the regressand for individual country, i, over period $\mathrm{t} ; X_{i t}$ is the exogenous regressors, country specific effects is $\mu_{i}$ while $v_{i t}$ is the remainder disturbance term. The theoretical application of GMM is justified by the introduction of lagged value of regressand as part of regressors which has led to the problem of autocorrelation. However, to overcome this econometric problem in the dynamic model, a number of empirical studies have suggested Arellano and Bond (1991) GMM estimator and Blundell and Bond (1998) system GMM estimator. The former differences the model in equation 4 purposely to get rid of the effects along with any time-invariant regressor as specified below:

$$
g_{i t}-g_{i t-1}=\sigma\left(g_{i t-1}-g_{i t-2}\right)+\beta\left(X_{i t}^{\prime}-X_{i t-1}^{\prime}\right)+\left(v_{i t}-v_{i t-1}\right)
$$

And it is assumed that $v_{i t}-v_{i t-1}$ follow first order moving average process (MA(1)). A problem with this estimator is that lagged levels are poor instruments for first differences if the variables are close to a random walk and hence system GMM es- 
timator. In addition to lagged levels of variables as instruments for equations in first differences, additional instruments can be brought to bear to increase efficiency.

$$
g_{i t}=\sigma z_{i t}^{\prime}+\beta X_{i t}^{\prime}+\mu_{i}+v_{i t} ; i=1, \ldots \ldots, n ; t=1, \ldots \ldots, T
$$

Where $z_{i t}$ is a vector of predetermined and endogenous covariates which may include the lag of $g_{i t}$ all of which may be correlated with the $\mu_{i t}$.

\section{Conclusion and Policy Recommendation}

This study considered the estimate three variants of the growth regression equation 3 using dynamic panel-data techniques of analysis. The results of these equations are presented in the respective tables as shown in the appendix. In the tables, the GDPK is the Gross Domestic Product per Capital; PGE is the Productive Government Expenditure, BD is the Budget Deficit, LAB is the human capital proxied by secondary school enrolment rate and GKF is the Gross Capital Formation. The empirical evidence provided in this study suggests that in sub-Saharan African countries, the productive government expenditure is not actually productive in relation with the economic growth over the period of 1980-2010 as shown by both Difference GMM and System GMM as shown in Table 5.

Contrary to the expectation but consistent with the relevant previous findings in the literature on the African economy, the results revealed negative effect of productive government expenditure on economic growth, as one percent increase in government expenditure leads to a significant magnitude decrease in economic growth in sub-Saharan Africa as shown in the dynamic panel data framework in Table 5 which takes into consideration both the source of the revenue and mode of expense. Also, contrary to the prediction of endogenous growth model, most of the productive government expenditures financed by non-distortionary taxation are counterproductive as revealed in Table 6 as the omission of government tax revenue does not statistically mitigate the negative effect of productive government expenditure on economic growth in sub-Saharan Africa. It is also revealed that the budget deficit is also negatively correlated with economic growth as shown in the Table 6 and Table 7. This is also in line with previous studies conducted within the African continent. This may be linked with the effect endemic corruption which is rampant among African politician. This study therefore, recommended that there must be good governance and necessary fiscal stimulus and discipline before African countries could realise and improve their economic development experience. 


\section{REFERENCES}

Alexander, W. (1990). Growth: some combined cross-sectional and time series evidence from OECD countries, Applied Economics, 22, 1197-1204.

Arellano, M. and Bond S. (1991). Some specification test for panel data Monte Carlo evidence and application to employment equation. Review of Economic Studies, 58,277,97.

Arellano, M. and Bover O. (1995). Another look at the instrumental variable estimation of error-components models. Journal of Econometrics, 68, 29, 52.

Barro, R. (1989). A cross-country study of growth, saving, and government, NBER Working Paper No. 2855.

Barro, R. (1990). Government spending in a simple model of endogenous growth, Journal of Political Economy: 98, s103-117.

Barro, R. (1991). Economic growth in a cross-section of countries. Quarterly Journal of Economics: $106,407-444$.

Barro, R. and Sala-i-Martin, X. (1992). Public finance in models of economic growth, Review of Economic Studies, 59, 645-661.

Barro, R. and Sala-i-Martin, X. (1995). Economic Growth, McGraw-Hill: New York.

Barro, R. and Sala-i-Martin X. (1992). Public finance in models of economic growth. Review of Economic Studies 59, 645, 6156

Barth, J.R. and Bradley, M. (1988). The impact of government spending on economic activity, mimeo, The National Chamber Foundation, Washington D.C.

Benos N. (2004). Fiscal Policy and Economic Growth: Empirical Evidence from OECD Countries. www.aueb.gr/crete2009/papers_recent/Benos.pdf

Bleany M., Gemmell N., and Kneller R. (1995). Economic growth (New York; McGraw-Hill)

Blundell, R. and Bond S. (1998). Innitial conditions and moment restrictions in dynamic panel data models,' Discussion Paper No. 97-07, University College London.

Cashin, P. (1995). Government spending, taxes and economic growth. IMF Staff Papers: 42, 237-269.

Castles, F. and Dowrick, S. (1990). The impact of government spending levels on medium-term economic growth in the OECD, 1960-85. Journal of Theoretical Politics: 2, 173-204.

Chamley, C. (1986). Optimal taxation of capital income in general equilibrium with infinite lives. Econometricia: 54, 607-622.

Devarajan, S., Swaroop, V. and Zou, H. (1996). The composition of public expenditure and economic growth, Journal of Monetary Economics: 37, 313-344.

Dowrick, S. (1992). Estimating the impact of government consumption on growth: Growth accounting and optimising models, unpublished; Canberra: Australian National University.

Easterly, W. and Rebelo, S. (1993). Fiscal policy and economic growth. Journal of Monetary Economics: $32,417-458$.

Engen, E. and Skinner, J. (1992). Fiscal policy and growth, NBER Working Paper No. 4223.

Grier, K. and Gordon T. (1989). An empirical analysis of cross-national economic growth, (1951 - 80) Journal of Monetary Economic, 24, 259-76.

Helms, L. (1985). The effect of state and local taxes on economic growth: a time series cross-section approach. Review of Economics and Statistics 67, 574-82.

Folster, S. and Henrekson, M. (1997). Growth and the public sector: A critique of the critics: IUI Working Paper Series No. 492. 
Glomm, G. and Ravikumar, B. (1994). Public investment in infrastructure in a simple growth model. Journal of Economic Dynamics and Control, 18, 1173-1187.

Glomm, G. and Ravikumar, B. (1997). Productive government expenditures and long-run growth. Journal of Economic Dynamics and Control, 21, 183-204.

Grier, K. and Tullock, G. (1989). An empirical analysis of cross-national economic growth (19511980). Journal of Monetary Economics: 24, 259-276.

Hansson, P. and Henrekson, M. (1994). A new framework for testing the effect of government spending on growth and productivity: Public Choice, 81, 381-401.

Helms, L. (1985). The effect of state and local taxes on economic growth: A time series-cross section approach, The Review of Economics and Statistics: 67, 574-582.

Hsieh, E. and Lai, K. (1994). Government spending and economic growth: the G7 experience. Applied Economics: 26, 535-42.

Jones, L., Manuelli, R. and Rossi, P. (1993). Optimal taxation in models of endogenous growth. Journal of Political Economy: 101, 485-519.

Judd, K. (1985). On the performance of patents, Econometrica: 53, 567-585.

King, R. and Rebelo, S. (1990). Public policy and economic growth: Developing neoclassical implications. Journal of Political Economy: 98, s126-151.

Kneller R., Bleaney M. and Gemmell N (1999). Fiscal Policy and growth: evidence from OECD countries. Journal of Public Economics: 74, 171-190.

Kneller R., Bleaney M. and Gemmell N. (1998). Growth, Public Policy and the Government Budget Constraint: Evidence from OECD Countries. Discussion Paper No. 98/14.

Koester, R. and Kormendi, R.C. (1989). Taxation, aggregate activity and economic growth: Cross-country evidence on some supply-side hypothesis. Economic Inquiry: 27, 367-386.

Kormendi, R.C. and Meguire, P.G. (1985). Macroeconomic determinants of growth: A cross-country study, Southern Economic Journal, 49, 783-792.

Korpi W. (1985). Economic growth and the welfare system: Leaky bucket or irrigation system?, European Sociological Review: 1, 97-118.

Landau, D. (1983). Government expenditure and economic growth. Southern Economic Journal: 10, 32-38.

Landau, D. (1985). Government expenditures and economic growth in the developed countries (19521976) Public Choice: 47, 459-477.

Landau, D. (1986). Government and economic growth in the less developed countries: An empirical study for (1960-1988) Economic Development and Cultural Change: 35, 35-75.

Levine, R. and Renelt, D. (1992). A sensitivity analysis of cross-country growth regressions, American Economic Review, 82, 942-63.

Lucas, R. (1990). Supply-side economics: An analytical review, Oxford Economic Papers: 42, 293-316.

Marsden, K. (1983). Links between taxes and economic growth: Some empirical evidence, World Bank Working Paper No. 605.

McCallum, J. and Blais, A. (1987). Government, special interest groups, and economic growth, Public Choice: $54,3-18$.

Mendoza, E., Milesi-Ferretti, G. and Asea, P. (1997). On the effectiveness of tax policy in altering long-run growth: Harberger's superneutrality conjecture, Journal of Public Economics: 66, 99-126.

Miller, S. and Russek, F. (1993). Fiscal structures and economic growth: International evidence", mimeo, University of Connecticut. 
Mofidi, A. and Stone, J. (1989). Do state and local taxes affect economic growth? Review of Economics and Statistics: 71, 686-691.

Nazmi, N. and Ramirez, M.D. (1997). Public and private investment and economic growth in Mexico, Contemporary Economic Policy, XV, January, 65-75.

Nehru, V., Swanson, E. and Dubey, A. (1995). A new database on human capital stock in developing and industrial countries: Sources, methodology and results, Journal of Development Economics, 46, 379-401.

Nordstrum, H. (1992). Studies in trade policy and economic growth, Stockholm University Monograph No. 20.

Persson, T. and Tabellini, G. (1994). Is inequality harmful for growth?. American Economic Review: 84, 600-621.

Ram, R. (1986). Government size and economic growth: A new framework and some evidence from cross-section and time series data, American Economic Review: 76, 191-203.

Romer, P. (1989a). What determines the rate of growth and technological change?. World Bank Working Papers.

Romer, P. (1989b). Capital accumulation in the theory of long-run growth, in: Barro R.(ed), Modern Business Cycle Theory, New York: Blackwell.

Romer, P. (1990). Human capital and growth: theory and evidence, Carnegie-Rochester Conference Series on Public Policy, 40, 47-57.

Sala-i-Martin, X. (1992). Public welfare and growth. Yale University, Economic Growth Centre Discussion Paper No. 666.

Skinner, J. (1987). Taxation and output growth: Evidence from African countries, NBER Working Paper No. 335.

Solow, R.M. (1956). A contribution to the theory of economic growth, Quarterly Journal of Economics, 71, 65-94.

Stokey, N. and Rebelo, S. (1995). Growth effects of flat-rate taxes Journal of Political Economy, 103, 510-550.

Swan, T.W. (1956) “Economic growth and capital accumulation”, Economic Record, 32, 334-361.

Weede, E. (1986), "Sectoral reallocation, distributional coalitions and the welfare state as determinants of economic growth rates in industrialised democracies", European Journal of Political Research, 14, 501-519.

Weede, E. (1991) “The impact of state power on economic growth rates in OECD countries”, Quality and Quantity, 25, 421-438.

Yi, K. and Kocherlakota, N. (1996) "Is there endogenous long-run growth? Evidence from the U.S. and the U.K.", Federal Reserve Bank of New York, 17, (December). 


\section{APPENDIX}

Table 5: Estimated result of all fiscal policy aggregated variables except budget deficit

\begin{tabular}{|c|c|c|c|c|}
\hline & $\begin{array}{c}\text { Diff GMM one step } \\
\text { (1) }\end{array}$ & $\begin{array}{c}\text { Diff GMM two step } \\
\text { (2) }\end{array}$ & $\begin{array}{l}\text { System GMM } \\
\text { one step } \\
\text { (3) }\end{array}$ & $\begin{array}{c}\text { System GMM } \\
\text { two step } \\
\text { (4) }\end{array}$ \\
\hline \multicolumn{5}{|c|}{ Dependent variable: $\boldsymbol{L o g}\left(\boldsymbol{G D P \boldsymbol { k } _ { i } )}\right.$} \\
\hline $\log \left(G D P K_{i t-i}\right)$ & $\begin{array}{c}1.59 * * * \\
{[3.64]} \\
(0.00)\end{array}$ & $\begin{array}{c}1.32 \\
{[4.68]} \\
(0.977)\end{array}$ & $\begin{array}{c}1.68 * * * \\
{[1.67]} \\
(0.00) \\
\end{array}$ & $\begin{array}{l}-8.39 \\
{[5.16]} \\
(0.87) \\
\end{array}$ \\
\hline $\log \left(P G E_{i i}\right)$ & $\begin{array}{c}-3.47 * * * \\
{[3.76]} \\
(0.00) \\
\end{array}$ & $\begin{array}{l}-3.23 \\
{[1.06]} \\
(0.976) \\
\end{array}$ & $\begin{array}{c}-5.28 * * * \\
{[2.30]} \\
(0.00) \\
\end{array}$ & $\begin{array}{c}-3.32 * * * \\
{[1.67]} \\
(0.04) \\
\end{array}$ \\
\hline $\log \left(N G R_{i}\right)$ & $\begin{array}{c}2.40 * * * \\
{[1.69]} \\
(0.00)\end{array}$ & $\begin{array}{l}-3.02 \\
{[7.38]} \\
(0.967)\end{array}$ & $\begin{array}{c}5.58 * * * \\
{[1.13]} \\
(0.00)\end{array}$ & $\begin{array}{c}3.48 * * * \\
{[1.69]} \\
(0.04)\end{array}$ \\
\hline $\log \left(B d_{i t}\right)$ & 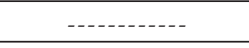 & - - - & - & - \\
\hline $\log \left(L a b_{i t}\right)$ & $\begin{array}{c}6.06^{* * * *} \\
{[1.34]} \\
(0.00)\end{array}$ & $\begin{array}{l}-5.52 \\
{[2.54]} \\
(0.983) \\
\end{array}$ & $\begin{array}{c}9.39 * * * \\
{[7.66]} \\
(0.00)\end{array}$ & $\begin{array}{c}9.74 \\
{[2.87]} \\
(0.00) \\
\end{array}$ \\
\hline $\log \left(G K F_{i t}\right)$ & $\begin{array}{c}2.19 * * * \\
{[3.69]} \\
(0.00)\end{array}$ & $\begin{array}{c}3.73 \\
{[1.11]} \\
(0.973) \\
\end{array}$ & $\begin{array}{c}-4.15 * * \\
{[2.30]} \\
(0.07)\end{array}$ & $\begin{array}{c}1.71 \\
{[1.67]} \\
(0.918)\end{array}$ \\
\hline Instrument & 342 & 342 & 148 & 148 \\
\hline No of Observation & 426 & 426 & 442 & 442 \\
\hline
\end{tabular}

Note:***,** denote significant at $1 \%$ and $5 \%$ respectively, [] denotes standard error, () is prob. of $\mathrm{z}$ value 
Table 6: Estimated result of fiscal policy aggregated variables except govt revenue

\begin{tabular}{|c|c|c|c|c|}
\hline & $\begin{array}{c}\text { Diff GMM one step } \\
\text { (1) }\end{array}$ & $\begin{array}{c}\text { Diff GMM two step } \\
\text { (2) }\end{array}$ & $\begin{array}{l}\text { System GMM } \\
\text { one step } \\
\text { (3) }\end{array}$ & $\begin{array}{c}\text { System GMM } \\
\text { two step } \\
(4) \\
\end{array}$ \\
\hline \multicolumn{5}{|c|}{ Dependent variable: $\boldsymbol{L} \boldsymbol{o g}\left(\boldsymbol{G D P \boldsymbol { k } _ { i t } )}\right.$} \\
\hline $\log \left(G D P K_{i t-i}\right)$ & $\begin{array}{c}-1.85 * * * \\
{[1.59]} \\
(0.00)\end{array}$ & $\begin{array}{c}1.90 \\
{[5.35]} \\
(0.997)\end{array}$ & $\begin{array}{c}1.20 * * * \\
{[3.06]} \\
(0.00)\end{array}$ & $\begin{array}{c}-3.10 * * * \\
{[4.29]} \\
(0.00)\end{array}$ \\
\hline $\log \left(P G E_{i}\right)$ & $\begin{array}{c}-2.18 * * * \\
{[1.06]} \\
(0.039) \\
\end{array}$ & $\begin{array}{c}1.69 \\
{[6.09]} \\
(0.998) \\
\end{array}$ & $\begin{array}{c}-4.08 * * * \\
{[2.74]} \\
(0.00) \\
\end{array}$ & $\begin{array}{c}-7.69 * * * \\
{[1.44]} \\
(0.00) \\
\end{array}$ \\
\hline $\log \left(N G R_{i}\right)$ & 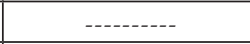 & 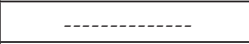 & 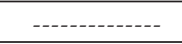 & 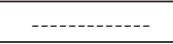 \\
\hline $\log \left(B d_{i t}\right)$ & $\begin{array}{l}-1.48 \\
{[7.38]} \\
(0.841) \\
\end{array}$ & $\begin{array}{l}-1.36 \\
{[1.59]} \\
(0.999) \\
\end{array}$ & $\begin{array}{c}-7.73 * * * \\
{[2.07]} \\
(0.00) \\
\end{array}$ & $\begin{array}{l}-6.30 \\
{[2.16]} \\
(0.00) \\
\end{array}$ \\
\hline $\log \left(\operatorname{Lab} b_{i t}\right)$ & $\begin{array}{l}5.11 * * \\
{[2.63]} \\
(0.052) \\
\end{array}$ & $\begin{array}{l}-1.17 \\
{[9.77]} \\
(0.999) \\
\end{array}$ & $\begin{array}{c}-4.44 * * * \\
{[1.41]} \\
(0.00) \\
\end{array}$ & $\begin{array}{c}-2.60 * * * \\
{[1.61]} \\
(0.00) \\
\end{array}$ \\
\hline $\log \left(G K F_{i t}\right)$ & $\begin{array}{c}5.52 \\
{[7.25]} \\
(0.446) \\
\end{array}$ & $\begin{array}{l}-9.86 \\
{[8.54]} \\
(0.999) \\
\end{array}$ & $\begin{array}{c}3.25 \\
{[4.22]} \\
(0.444) \\
\end{array}$ & $\begin{array}{l}-2.37 * * \\
{[1.32]} \\
(0.072) \\
\end{array}$ \\
\hline Observation & 426 & 426 & 442 & 442 \\
\hline Instrument & 342 & 342 & 148 & 148 \\
\hline
\end{tabular}

Note:***,** denote significant at $1 \%$ and $5 \%$ respectively, [] denotes standard error, () is prob. of $\mathrm{z}$ value 
Table 7: Estimated result of fiscal policy aggregated variables except govt expenditure

\begin{tabular}{|c|c|c|c|c|}
\hline & $\begin{array}{c}\text { Diff GMM one step } \\
\text { (1) }\end{array}$ & $\begin{array}{c}\text { Diff GMM two step } \\
\text { (2) }\end{array}$ & $\begin{array}{l}\text { System GMM } \\
\text { one step } \\
\text { (3) }\end{array}$ & $\begin{array}{c}\text { System GMM } \\
\text { two step } \\
\text { (4) }\end{array}$ \\
\hline \multicolumn{5}{|c|}{ Dependent variable: $\boldsymbol{L} \boldsymbol{o g}\left(\boldsymbol{G D P k _ { i t } )}\right.$} \\
\hline $\log \left(G D P K_{i t-i}\right)$ & $\begin{array}{l}1.08 * * * \\
{[7.11]} \\
(0.00)\end{array}$ & $\begin{array}{l}-1.35 \\
{[3.04]} \\
(0.96) \\
\end{array}$ & $\begin{array}{c}6.74 * * * \\
{[3.08]} \\
(0.00)\end{array}$ & $\begin{array}{c}6.60 * * * \\
{[1.97]} \\
(0.00) \\
\end{array}$ \\
\hline $\log \left(P G E_{i}\right)$ & --.----- & --.----- & --------- & - ----o---- \\
\hline $\log \left(N G R_{i i}\right)$ & $\begin{array}{l}-3.13 \\
{[4.74]} \\
(0.509)\end{array}$ & $\begin{array}{c}2.16 \\
{[9.70]} \\
(0.98) \\
\end{array}$ & $\begin{array}{c}-2.38 * * * \\
{[2.75]} \\
(0.00)\end{array}$ & $\begin{array}{c}3.55 \\
{[6.91]} \\
(0.67) \\
\end{array}$ \\
\hline $\log \left(B d_{i t}\right)$ & $\begin{array}{l}-1.48 \\
{[7.38]} \\
(0.84) \\
\end{array}$ & $\begin{array}{c}1.54 \\
{[8.10]} \\
(0.985) \\
\end{array}$ & $\begin{array}{c}-5.92 * * * \\
{[4.24]} \\
(0.00)\end{array}$ & $\begin{array}{l}-2.33 \\
{[1.73]} \\
(0.178) \\
\end{array}$ \\
\hline $\log \left(\operatorname{Lab} b_{i t}\right)$ & $\begin{array}{c}5.11 \\
{[2.63]} \\
(0.841)\end{array}$ & $\begin{array}{l}-2.42 \\
{[5.32]} \\
(0.996)\end{array}$ & $\begin{array}{c}7.23 * * * \\
{[1.41]} \\
(0.00)\end{array}$ & $\begin{array}{c}2.91 \\
{[3.55]} \\
(0.412)\end{array}$ \\
\hline $\log \left(G K F_{i t}\right)$ & $\begin{array}{c}5.52 \\
{[7.25]} \\
(0.446) \\
\end{array}$ & $\begin{array}{l}-1.54 \\
{[6.45]} \\
(0.998) \\
\end{array}$ & $\begin{array}{c}3.10 \\
{[4.24]} \\
(0.464) \\
\end{array}$ & $\begin{array}{l}-2.30 \\
{[1.55]} \\
(0.882)\end{array}$ \\
\hline Observation & 426 & 426 & 442 & 442 \\
\hline Instrument & 342 & 342 & 148 & 148 \\
\hline
\end{tabular}

Note:****** denote significant at $1 \%$ and $5 \%$ respectively, [] denotes standard error, () is prob. of $\mathrm{z}$ value 